\title{
Research On Globe-cone Joint Assembly Of Spacecraft Propulsion System
}

\author{
Yanlei Zhang ${ }^{1,2, a}$, Feikai Zhang ${ }^{3}$, Yuchuan Bian ${ }^{1,2}$, Yougao Fan ${ }^{1,2}$ and Kai $\mathrm{Xu}^{1,2}$ \\ ${ }^{1}$ Beijing Institute of Spacecraft Environment Engineering, 100094, Beijing \\ ${ }^{2}$ Beijing Engineering Research Center of the Intelligent Assembly Technology and Equipment for Aerospace \\ Product, 100094, Beijing \\ ${ }^{3}$ Beijing Institute of Technology, 100081, Beijing
}

\begin{abstract}
As one of the key step of spacecraft propulsion system assembly, globe-cone joint assembly determines the spacecraft's high reliability and long life on orbit. Through test, simulation analysis and calculation on sealing mechanism of globe-cone joint, in this paper, we make sure the relationship of the tightening torque and globe-cone joint contact stress and contact area, and obtain assembly torque range of different specifications of globe-cone joint, which gives necessary reference for mechanical environment verification.
\end{abstract}

\section{Background}

Propulsion system is the most important subsystem of spacecraft, its performance directly influence control precision, reliability and life on orbit. Generally, the propulsion system consists of thruster, propellant tank, latching valve, fill/drain valve, pressure sensor, filter, etc. The propellant is transported by been pushed in pipeline, so, propulsion system has high pressure inside. To insure sealing reliability, the way of welding is adopted in pipeline connecting, while globe-cone joint is adopted between pipeline and thruster, which have cone surface on thruster and globe surface on pipeline, and this way connecting is widely used on DFH(Short for Dongfanghong)-3 satellite platform, DFH-4 satellite platform, remote sensing satellite platform and navigation satellite platform. The tightening torque is one of the factors to affect the globe-cone joint assembly performance. Through test and simulation, this paper gives the minimum and maximum torque value during certain type of globe-cone joint assembly, which provides necessary parameters for further research to satisfy mechanical environment.

\section{Globe-cone joint sealing mechanism and research approach}

The globe-cone joint seal structure belongs to the type of metal contact and static seal, through giving external force, it forms a compact seal surface after incessant pressure. The force diagram is shown in figure 1 .

${ }^{\mathrm{a} C}$ Corresponding author:yan_hust@163.com 


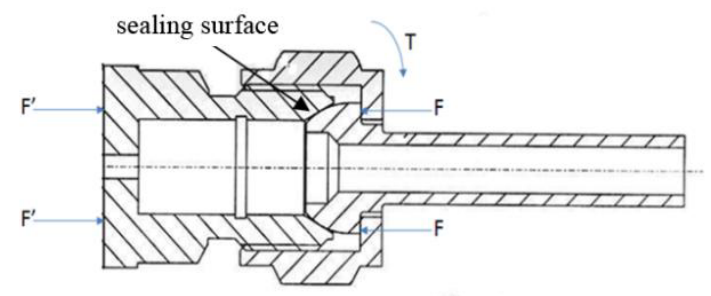

Figure 1. Globe-cone joint structure force diagram

Figure 2 gives the failure tree of the globe-cone joint seal leak rate. It can be seen that the factors to affect seal quality are divided to two kinds by property: seal surface machining factor and assembly process factor. The machining factor refers to the surface of the sealing surface roughness, parts with surface shape error, which is mainly embodied in the globe-cone geometry. Assembly process factor refers to the comprehensive factor including technique, operation experience, operating environment and other factors, mainly reflected in the neutral adjustment, globe-cone relative motion control, tightening torque, tightening stress control and redundancy control etc. The control of tightening torque is the most critical factor in the practice.

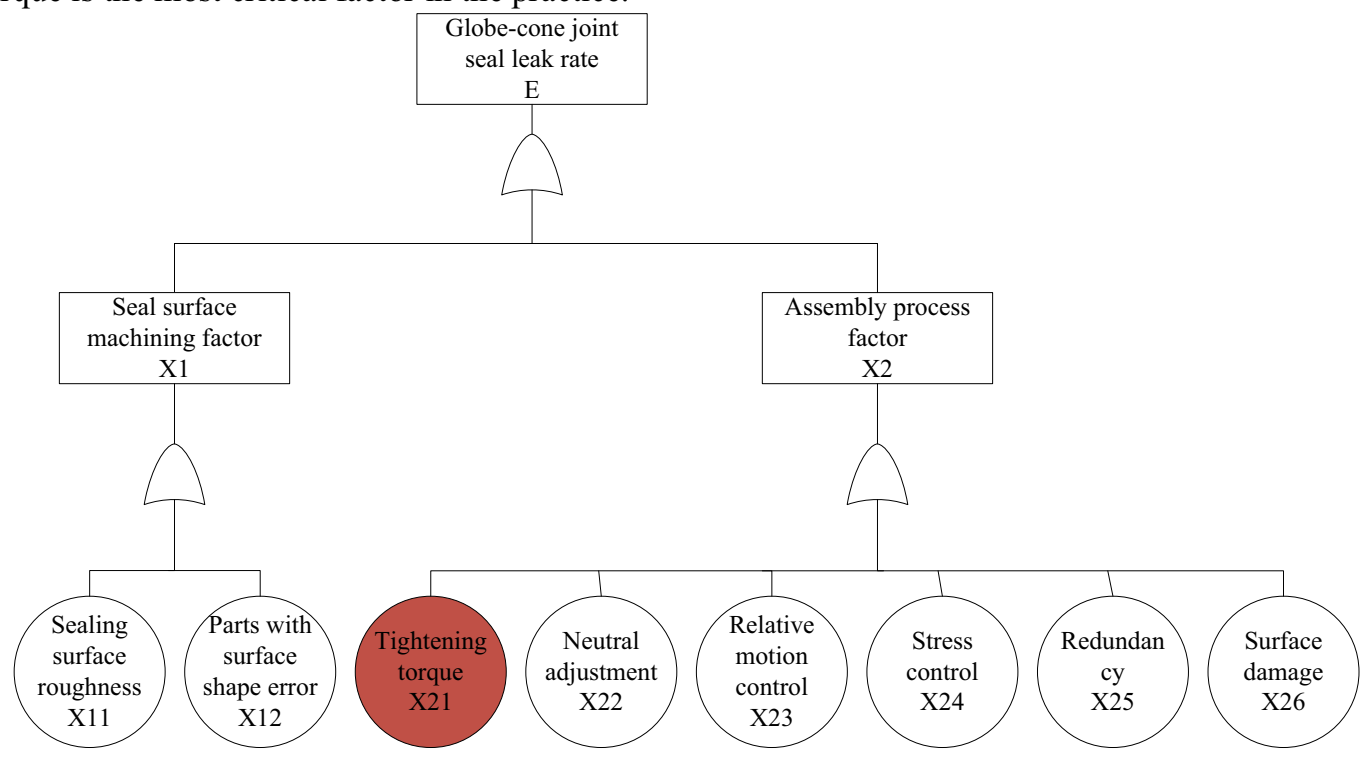

Figure 2. Effect of globe-cone joint structure factors

The research flow of assembling tightening torque shown in Figure 3 is presented.

1. Using experimental measurements to obtain the relationship of globe-cone joint between pretightening force and torque assembly method, and the coefficient of friction between the friction pair measured at the same time, to be the parameters of finite element analysis.

2. Using the method of finite element simulation of globe-cone joint structure model and the relationship between the structure of the globe-cone contact state and pre-tightening force.

3. Using the method of judging the relationship between the sealing performance and contact state. 


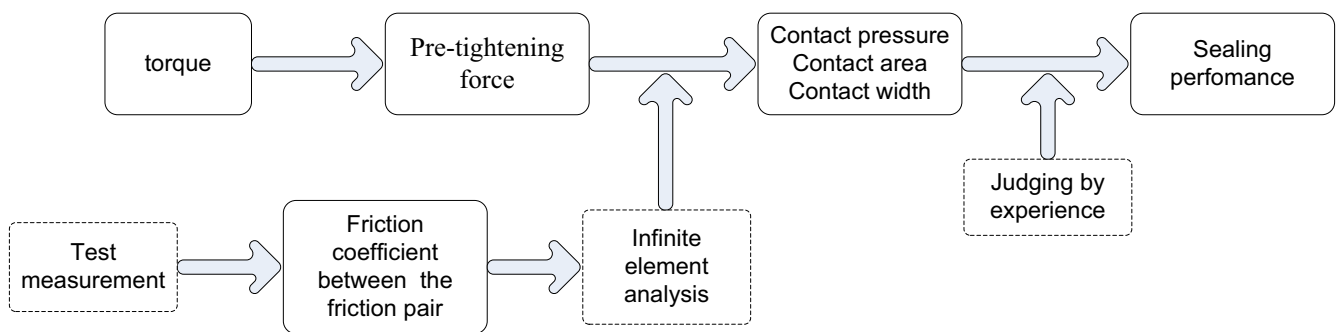

Figure 3. Assembling tightening torque research flow

\section{Research on relationship between torque and pre-tightening force}

There are two main methods to obtain the globe-cone assembly and other similar cone sealing structure torsion relationship: by theoretical calculation [1] and measured by experiment [2].

Including the ball screw thread structure head sealing structure, in the assembly process, the applied around the axial torque tightening torque, the torque generated by the pre-tightening force in the axial internal thread structure; therefore the pre-tightening force of the screw thread structure will be affected by their assembly tightening torque influence.

The relationship between the axial torque and the pre-tightening force is known as the formula 1 .

$$
T=K F d
$$

In the formula, $\mathrm{T}$ is the tightening torque, $\mathrm{K}$ is the torque coefficient, $\mathrm{F}$ is the pre-tightening force, and $\mathrm{d}$ is the nominal diameter of the thread.

The torsion coefficient $\mathrm{K}$ can be expressed as a series of calculations:

$$
\mathrm{K}=\frac{1}{2}\left[1.15 \mu_{\mathrm{s}}+\tan \beta+\mathrm{d}_{\mathrm{w}} \mu_{\mathrm{w}} / \mathrm{d}+\mathrm{D}_{\mathrm{MP}} \mu_{\mathrm{N}} /(\mathrm{d} \cdot \cos \alpha)\right]
$$

$F$ is preload, $\alpha$ is cone angle, $D_{M P}$ is globe-cone contact surface equivalent diameter, $\mu_{N}$ is globecone friction coefficient, $d_{W}$ is the support surface equivalent diameter, $\mu_{w}$ is the support surface friction coefficient, $\mu_{s}$ is the thread friction coefficient, $\beta$ is helix angle.

The friction coefficient of the globe-cone structure is unknown and it changes in a certain range, related to materials, surface contact friction coefficient and substrate surface morphology and other factors. At the same time, equivalent diameter of the supporting surface and equivalent globe and cone contact areas are difficult to be accurately determined. Therefore, the torque coefficient cannot be well matched with the actual value, and the formula method is not practical.

Through the method of testing the relationship of the globe-cone torsion, we can obtain accurate measurement torsion of globe-cone joint structure, and also can measure the coefficient of each friction pair. This method is shown in Figure 4, the out nut is cut from the non-screw thread section, turned the globe-cone inner force to out nut sections outer force, for convenient measurement. 


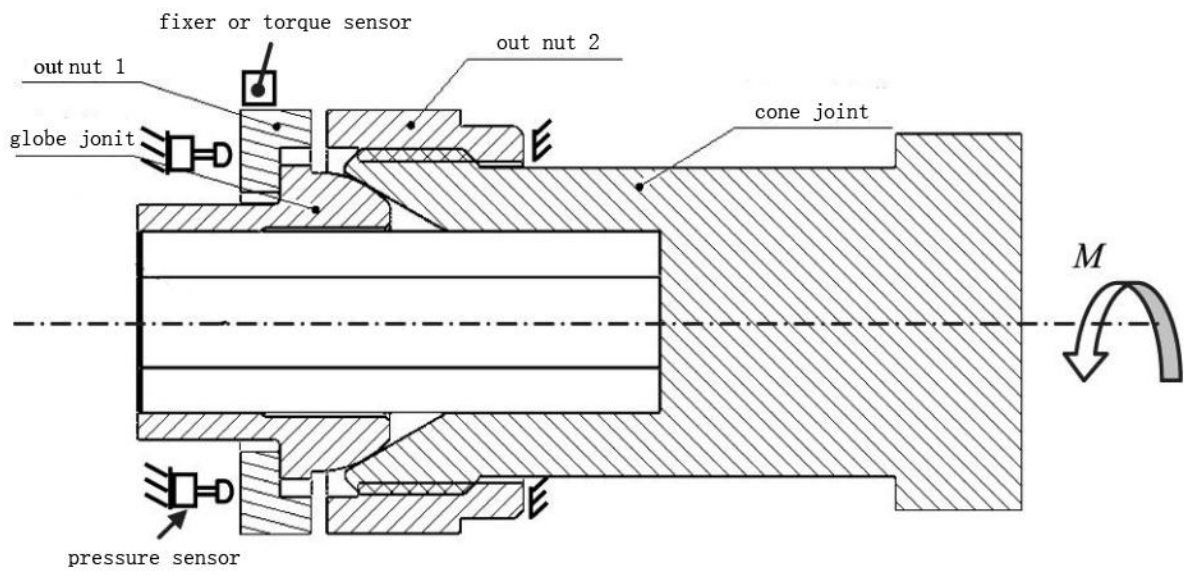

Figure 4. The globe-cone structure torsion relationship test principle

Take the type of 60-SR5.5-4 globe-cone joint sealing structure as an example, the detailed test results are shown in table 1.

Table 1. The type of 60-SR5.5-4 test results

\begin{tabular}{|l|l|l|l|l|l|l|l|l|l|}
\hline No. & $\mathbf{M a x F} / \mathbf{k N}$ & $\mathbf{M a x T} / \mathbf{N m}$ & $\mathbf{T}_{\text {tot }} / \mathbf{N m}$ & $\mathbf{T}_{\text {th }} / \mathbf{N m}$ & $\mathbf{T}_{\mathbf{b}} / \mathbf{N m}$ & $\mathbf{K}$ & $\boldsymbol{\mu}_{\text {tot }}$ & $\boldsymbol{\mu}_{\text {th }}$ & $\boldsymbol{\mu}_{\mathbf{b}}$ \\
\hline 1 & 5.42 & 30.00 & 16.14 & 4.76 & 11.38 & 0.38 & 0.43 & 0.47 & 0.35 \\
\hline 2 & 5.52 & 30.00 & 15.97 & 4.46 & 11.51 & 0.38 & 0.42 & 0.48 & 0.33 \\
\hline 3 & 5.07 & 30.00 & 16.55 & 6.68 & 9.87 & 0.39 & 0.44 & 0.41 & 0.49 \\
\hline
\end{tabular}

Note: Max T is the pre-tightening force(the test stop condition, namely $30 \mathrm{Nm}$ ); $T_{t o}, T_{t h}, T_{b}$ respectively is the total torque, thread torque and support surface torque; $K$ is torque coefficient; $\mu_{t o t}, \mu_{t h}, \mu_{b}$, is respectively the total friction coefficient, thread friction coefficient and support surface friction coefficient.

From table 1, we can see that the torque coefficient $\mathrm{K}$ of three groups is basically the same, respectively $0.38,0.38$ and 0.39 . There are some differences in thread friction coefficient and support surface friction coefficient. The causes of this phenomenon may be relative to the sliding surface which is more than one.

\section{Finite element analysis of pre-tightening force and contact state}

The material of different specifications of sealing globe-cone joint is different. Take the type of 60SR5.5-4 for example, globe-cone seal structure material are titanium alloy TC4 and stainless steel $1 \mathrm{Cr} 18 \mathrm{Ni9Ti}$. In finite element analysis of the globe-cone sealing structure assembly, the bilinear isotropic hardening model is taken to define the properties of the two materials. The materials and their properties are shown in Table 2.

Table 2. Materials of globe-cone joint sealing structure

\begin{tabular}{|l|l|l|l|}
\hline Specifications/Properties & Cone material & Outer nut material & Globe material \\
\hline $60-$ SR5.5-4 & stainless steel 1Cr18Ni9Ti & titanium alloy TC4 & titanium alloy TC4 \\
\hline Young's modulus & $206 \mathrm{GPa}$ & $109 \mathrm{GPa}$ & $109 \mathrm{GPa}$ \\
\hline Poisson ratio & 0.3 & 0.34 & 0.34 \\
\hline Yield strength & $205 \mathrm{MPa}$ & $840 \mathrm{MPa}$ & $840 \mathrm{MPa}$ \\
\hline Tangent modulus & $6.8 \mathrm{GPa}$ & $2 \mathrm{GPa}$ & $2 \mathrm{GPa}$ \\
\hline
\end{tabular}

In order to get the accurate result, structured grid is used to partition the model. The globe-cone sealing structure of the model is not a common rule model, and the contact surface is related to plastic deformation, therefore the model has nonlinear material and structure. The element type is SOLID185 unit with 3D 8-node, the number of grid model is 338,000, as shown in figure 5. 


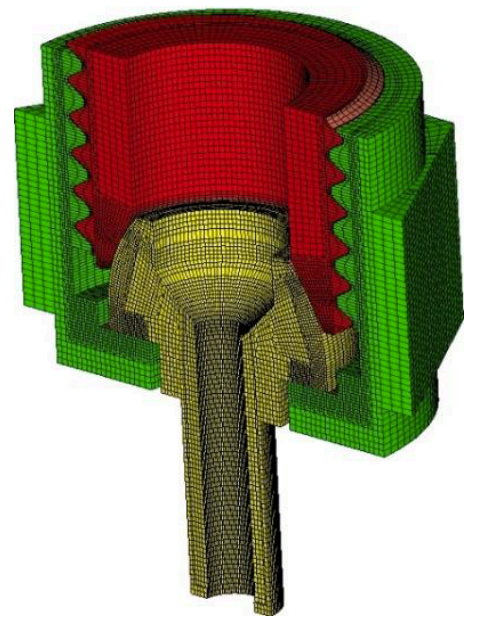

Figure 5. Grid density of globe-cone joint structure

In the model of the project, the three parts are all elastic bodies, and the final state of the contact pair is surface contact. So their contact surfaces are defined as surface contact and elastic-elastic contact.

Based on the grid density model test, the contact interface parameter setting and the boundary conditions imposed, we set up the globe-cone sealing structure assembly simulation of the finite element model as shown in figure 6. Globe-cone joint sealing structure contact friction coefficient can be found in table 1 .

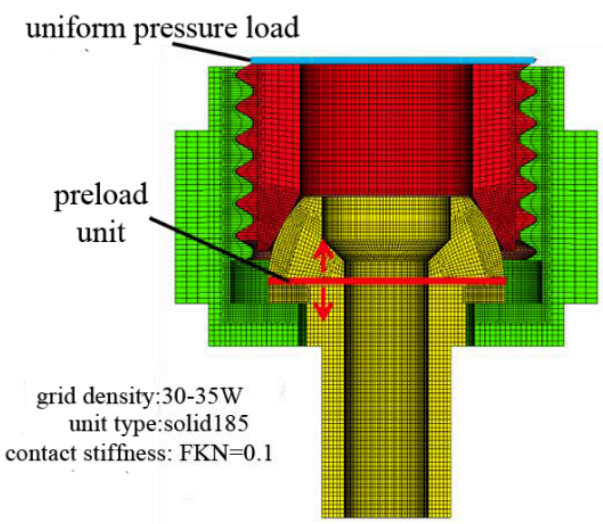

Figure 6. Assembly simulation of the finite element model

On the finite element model simulation analysis, we can get the globe-cone sealing structure of the equivalent stress (Von-mise stress), contact stress, change of base area with pre-tightening force.

Table 3 shows the equivalent stress of the sealing structure under increasing preload. Comparison of four images, we can see that, with the increase of pre-tightening force, equivalent stress increases, and the stress concentration point exits in the maximum diameter of contact surface circle between the out nut and globe.

Table 3. Transformation of contact stress with increasing preload

\begin{tabular}{c|l|l} 
& Equivalent stress distribution & contact stress distribution \\
\hline
\end{tabular}




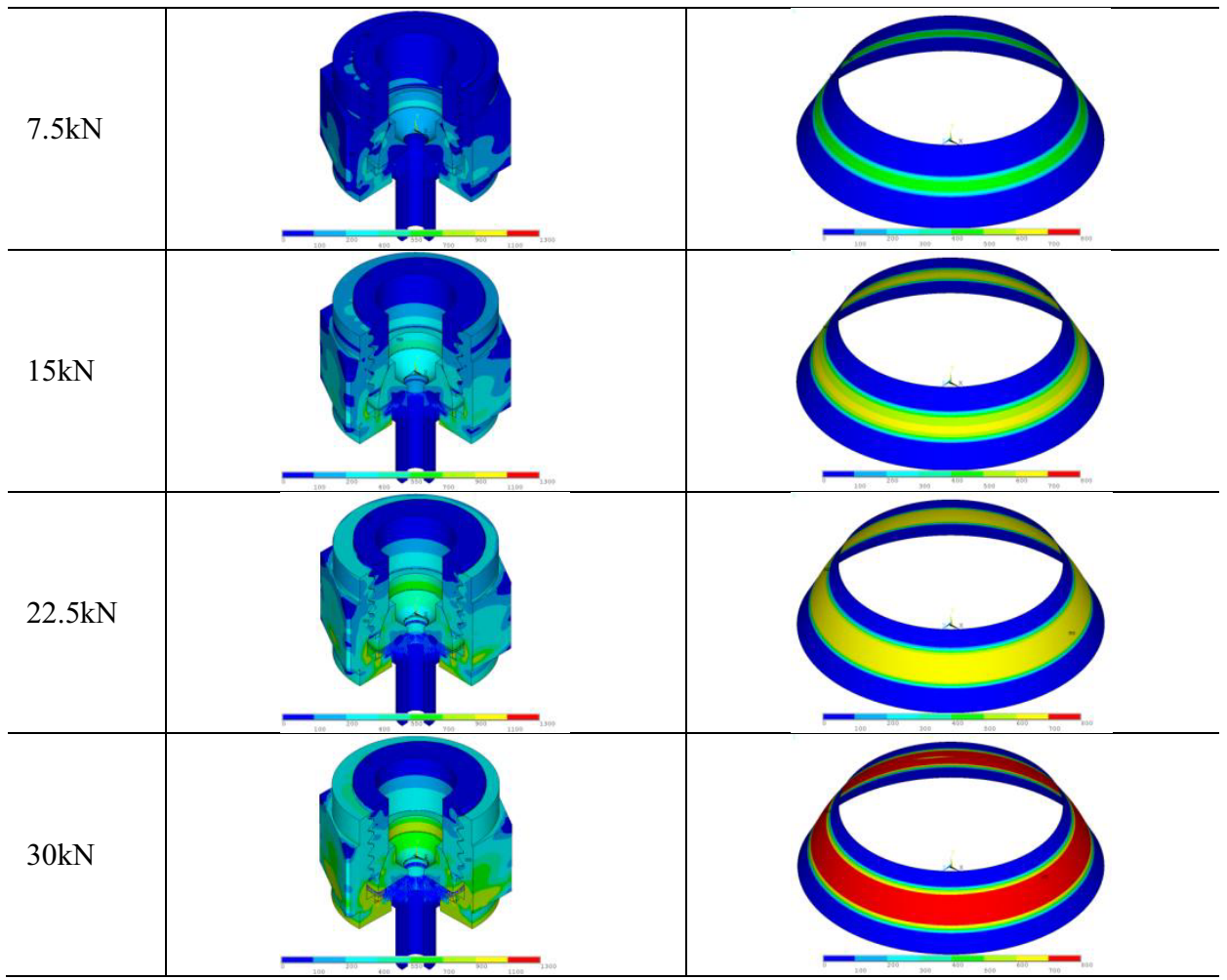

\section{Study on the relationship between contact state and sealing}

In this project, the average contact stress and the contact width are used to determine the preload range.

\subsection{Contact pressure criterion}

On average contact pressure, the overall judgment criterion is to ensure that the average contact pressure of the sealing surface is greater than the yield strength of the materials [3].

\subsection{Contact area criterion}

On the contact area, it needs the contact analysis to judge the contact width required, to ensure the sealing performance of the contact area. Based on the commonly used leakage model A Roth, this project has improved, the cone contour surface is simplified as a plane and the globe surface is simplified as a cylinder [4-5], whose radius is the radius of the globe. The improved model is shown in figure 7. 


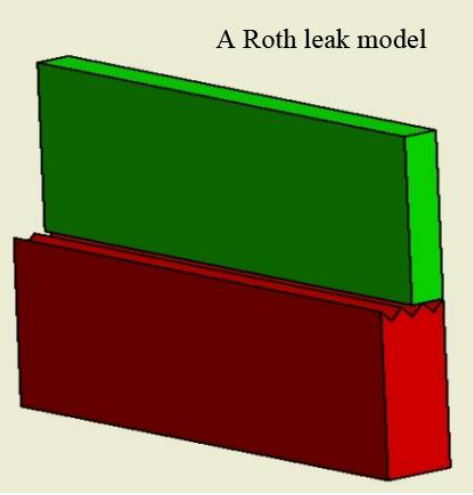

(a)

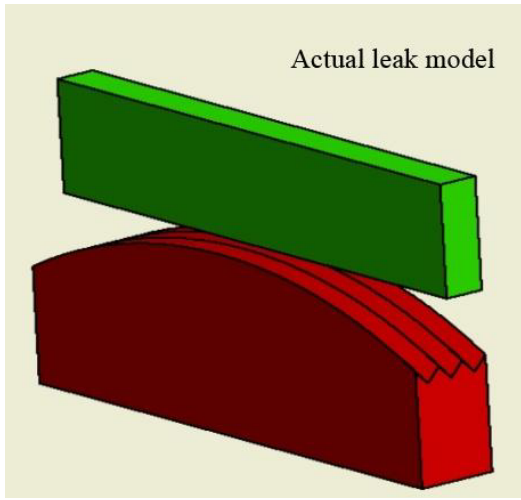

(b)

Figure 7. Microscopic contact model

Because the actual micro contact model is a symmetrical structure in the flow direction, and a triangular wave periodic structure perpendicular to the flow direction [6-7], so in the finite element model, we select a single flow channel perpendicular to the flow direction and half the channel along the flow direction to analysis. The solid45 unit is chosen as the cell type of the model, which is divided into a finite element model with 325,800 structured meshes. The triangle wave surface is defined as the contact surface, and the contact element type is Contact174. The corresponding ideal plane is defined as the target plane, and the target cell type is Target170. Because of the special model, the symmetry constraint is imposed on the plane and the symmetry constraint is applied in front and behind of the plane. At the same time, a uniform pressure load is applied on the top surface, and a static constraint at the bottom, as shown in figure 8 .

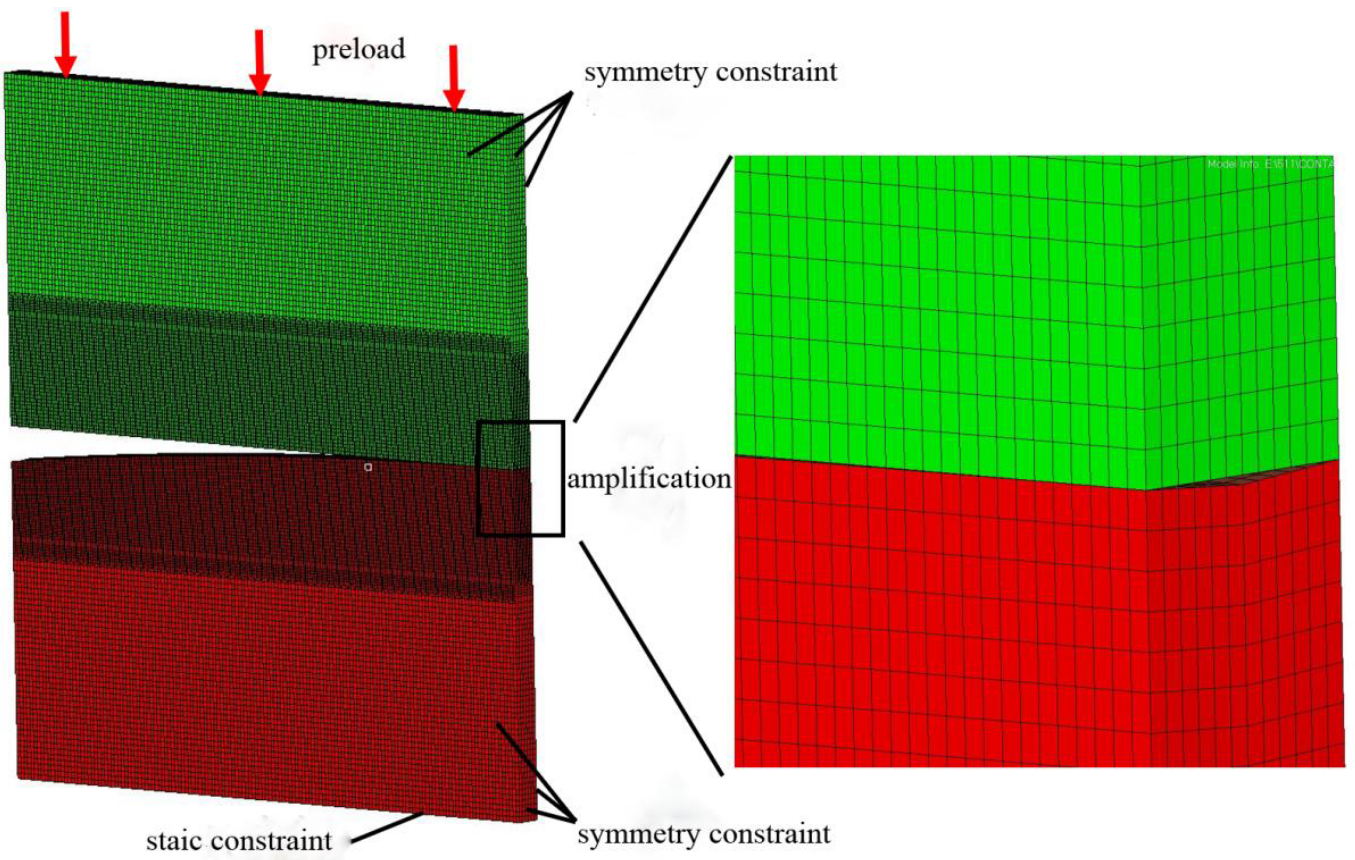

Figure 8. Leakage channel cross section and microscopic contact model

The evolution of the simulation calculation of globe-cone joint sealing structure and micro contact width of the contact area is shown in figure 9. The contact area increases with the gradual increase of the contact width, and the width of the channel leakage begin to decrease when the contact width 
increases to a certain value, finally, the isolated contact areas are connected to each other and leak channel is completely blocked.

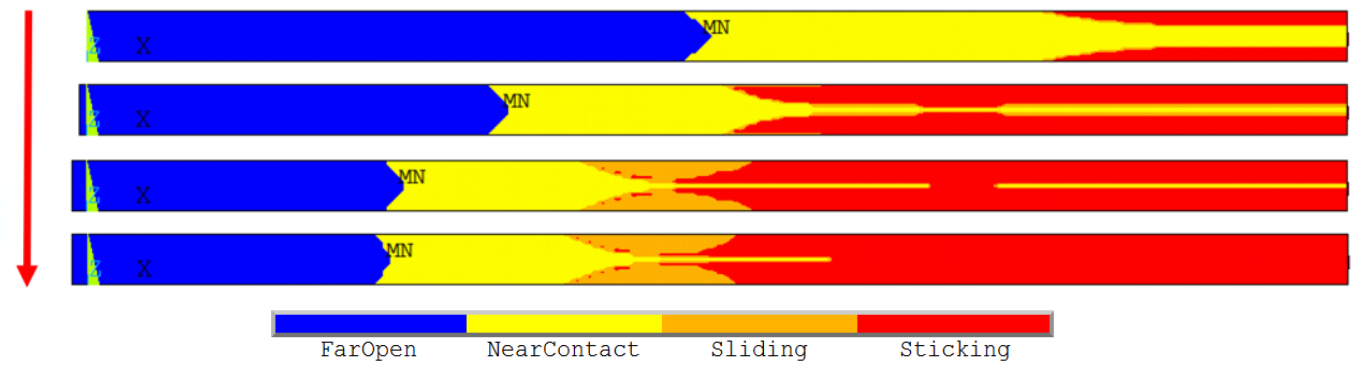

Figure 9. The evolution of micro contact and width the contact area

According to the approximate solution equation 3 of the contact area, we can get the globe-cone contact area standard as shown in table 4.

$$
\mathrm{A}=\sqrt{3} \pi \mathrm{lr}
$$

A is the sealing interface contact area, 1 is contact width, $r$ is radius of the globe.

Table 4. Globe-cone contact area standard

\begin{tabular}{|l|l|l|}
\hline Specification & Contact width $/ \mathrm{mm}$ & Contact area $/ \mathrm{mm}^{2}$ \\
\hline $60-$ SR5.5-4 & 0.714 & 21.36 \\
\hline
\end{tabular}

\section{Pre-tightening force judgment and conclusion}

\subsection{Determination of lower limit of pre-tightening force}

In order to satisfy the ideal sealing result, the average contact pressure preload should be up to $205 \mathrm{MPa}$ to seal the interface, at the same time, the contact area should be more than $21.36 \mathrm{~mm}^{2}$. As shown in Figure 10, the contact area and contact press change with the pre-tightening force, we choose the larger pre-tightening force as the lower limit of preload, which is $4.34 \mathrm{kN}$.

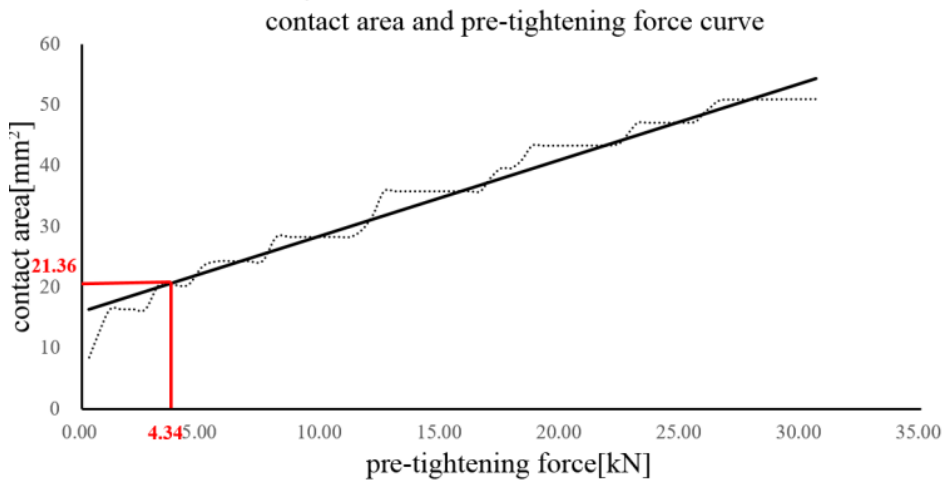




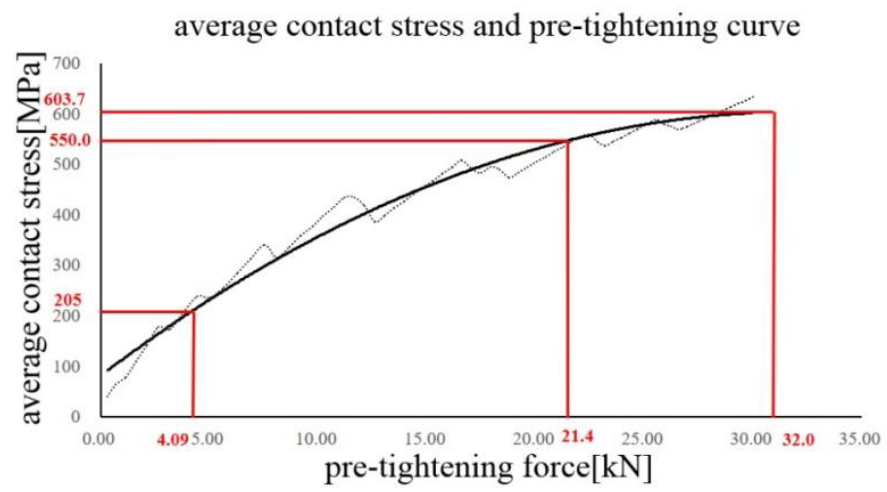

Figure 10. Contact area and press change with pre-tightening force

\subsection{Determination of upper limit of pre-tightening force}

Also from Figure 10, when the average contact stress is greater than $603.7 \mathrm{MPa}$, the average contact stress decreases with the pre-tightening force. When the pre-tightening force comes to $21.4 \mathrm{kN}$, the average contact stress reaches the ultimate strength of the structure material of 550MPa.

For the thread structure (M14), the maximum tightening torque applied on the part of the screw should ensure that the average stress is not greater than $70 \%$ of the yield strength, the average stress on the screw part can be got from formula 4. In 60-SR5.5-4 globe-cone structure, D1 is $12.376 \mathrm{~mm}, \mathrm{~d} 1$ is $8 \mathrm{~mm}$, and $\sigma_{\mathrm{s}}$ is $205 \mathrm{MPa}$. It can be calculated that $\mathrm{F}$ is no more than $10.0 \mathrm{kN}$.

$$
\sigma=F /\left(\frac{\pi}{4}\left(D_{1}-d_{1}\right)\right) \leq 0.7 \sigma_{s}
$$

Based on the above conditions, it can be concluded that the upper limit of pre-tightening force for sealing condition is $10.0 \mathrm{kN}$.

\subsection{Conclusion}

According to 3rd section of the globe-cone assembly torsional tension test results, the torque coefficient $\mathrm{K}$ is 0.38 , and according to the formula 1 , we can calculate the tightening torque range of the sealing structure is $23.1-53.2 \mathrm{Nm}$.

With the same analysis and calculation method, we can obtain the tightening torque range for other type of globe-cone joint widely used on spacecraft at present, which is shown in table 5.

Table 5. Tightening force of different joint structure

\begin{tabular}{|l|l|l|l|}
\hline $\begin{array}{l}\text { Thruster } \\
\text { specifications }\end{array}$ & Specifications & $\begin{array}{l}\text { Pre-tightening } \\
\text { force/kN }\end{array}$ & $\begin{array}{l}\text { Tightening } \\
\text { torque/Nm }\end{array}$ \\
\hline $1 \mathrm{~N}, 5 \mathrm{~N}, 20 \mathrm{~N}$ & $60-\mathrm{SR} 5.5-6$ & $4.75-11.1$ & $24.6-57.5$ \\
\hline $10 \mathrm{~N}$ & $60-\mathrm{SR} 5.5-4$ & $4.34-10.0$ & $23.1-53.2$ \\
\hline $490 \mathrm{~N}$ & $60-\mathrm{SR} 7.3-8$ & $6.97-20.1$ & $47.7-137.5$ \\
\hline E-propulsion & $60-\mathrm{SR} 2.75-4$ & $2.29-4.94$ & $7.15-15.4$ \\
\hline
\end{tabular}

Besides the simulation analysis and calculation of the torque range, it needs further research to verify the torque range under mechanical environment, and to narrow the range.

\section{References}


1. Zhou Xin, Pang Hewei, Liu Hongyang, Yan Shaoguang, Influence analysis of assembly error on seal status of spherical seal structure, J. Spacecraft Engineering. 2005, (04) 35-39.

2. Liu Kai, Li Qiang, Xie Zubao, Study on control of pipe joint tightening torque based on torque test, J. Quality and Reliability. 2014, (5) 16-19.

3. H.H. Buchter. Industrial sealing technology [ M] .Beijing: Chemical Industry Press, 1988.

4. Robbe-Valloire, F., and Prat, M., 2008, "A Model for Face-Turned Surface Microgeometry: Application to the Analysis of Metallic Static Seals," Wear, 264(11), pp. 980-989.

5. Zhang F, Liu J, Ding X, Yang Z. An Approach to Calculate Leak Channels and Leak Rates Between Metallic Sealing Surfaces. ASME. J. Tribol. 2016;139(1):011708-011708-11.

6. Zhou Xin, Pang Hewei, Liu Hongyang, Yan Shaoguang. Nonlinear contact analysis of globe-cone connection structure [J]. Spacecraft Environment Engineering,2005,(04):211-214.

7. Wang Xiaogang, Zhang Fangxiao, Huang Peng, Design of cone-head \& cone-hole pipeline cone sealing structure based on ANSYS[J]. Machinery,2009,(08):37-40. 